\title{
Termografia: ferramenta auxiliar na pesquisa de materiais e no design de produtos
}

\author{
Silva, Júlio César Riccó Plácido ${ }^{\mathrm{a}}$; Mira, Maria do Rosário Gonçalves ${ }^{\mathrm{b}}$ \\ a Doutorando (Design e Arquitetura,Universidade de São Paulo, Brasil) julioricco@usp.br, \\ ${ }^{\text {b }}$ Doutoranda (Design e Arquitetura,Universidade de São Paulo, Brasil) mariarosariomira@usp.br,
}

\begin{abstract}
Resumo
Termografia é um procedimento de medição passiva e sem contato, que registra a distribuição de temperatura na superficie de um material, constituindo-se como um aporte para análises de condutividade térmica, além de contribuir para os estudos a respeito da percepção tátil. Este equipamento possibilita ao designer reunir recursos adicionais ao procedimento de mapeamento da relação de percepção, permitindo uma prospecção aprofundada e interior da matéria em estudo. O equipamento gera imagens a partir de um espectro de cores, exprimindo variações de temperatura, destacando detalhes que não são perceptiveis a olho nu e permitindo a visualização de áreas e variações de emissão de calor da superficie do objeto. Tais dados se tornam diferenciais e indutivos para o processo de projeto. Considerando a importância da termografia, este artigo direciona seus estudos para validar esse instrumento auxiliar de coleta de dados, que permite compreender a percepção a partir da interação do usuário com polímeros, identificando qualidades inerentes a cada tipo de material e indicando novas possibilidades e alternativas de uso. Plásticos ou polímeros não são em si uma entidade única de material, mas muitas categorias com qualidades e propriedades especificas, representando uma classe a parte entre as modalidades de materiais. Usuários utilizam-se da interação tátil para avaliar essas qualidades. Mapear essa interação por meio da termografia pode fornecer dados relevantes para designers no desenvolvimento de produtos. Para a coleta de dados foi utilizada a termo-câmera Flir Ex6 com detector de matriz de plano focal (FPA), microbolómetro não refrigerado, com gama espectral 7,5-13 $\mu$ m. Para o corpo de amostras foram selecionados os polimeros: PP, PMMA, Poliestireno, PU, PVC expandido e rigido, $P A$ e PC em tamanho de $80 \times 80 \mathrm{~mm}$ e espessuras variando entre 3 e $5 \mathrm{~mm}$. A variação de espessuras se deve ao padrão de mercado. Participaram do estudo 10 pessoas, homens e mulheres, de idades variadas, em amostra não estratificada. Pretendeu-se com o estudo confirmar a termografia como uma ferramenta auxiliar de investigação para melhor interpretação das variáveis térmicas dos polímeros e averiguar tanto a sensibilidade de contato como a condutividade de cada material e se o fator de condutividade térmica altera a percepção de suas qualidades pelos usuários.
\end{abstract}

Palavras-chaves: termografia, termograma, percepção de materiais, design, polimeros. 


\begin{abstract}
Thermography is a passive and contactless measuring procedure, which registers the temperature distribution along the surface of a material, constituting as a standing to thermal conductivity analysis, as well as a contribution to studies of the tactile perception. This equipment enables the designer to gather additional resources to the mapping procedure concerning the relation of perception, allowing an in-depth and inner exploration of the studied material. The device generates images from a color spectrum, expressing variations in temperature, and highlighting details that are not visible to the naked eye, allowing also the viewing of heat areas and the variations of heat emission along the surface of the object. Such data become differential and inductive to the design process. Considering the importance of thermography, this article focuses its studies to validate this instrument of data collection, which allows us to understand the perception from the user's interaction with polymers, identifying qualities inherent in each type of material and indicating new possibilities and usage alternatives. Plastics or polymers are not in themselves a single material entity, but they comprise many categories with specific qualities and properties, representing a peculiar class among the modalities of materials. Users resort the tactile interaction to assess these qualities. The mapping of this interaction through thermography can provide relevant data to designers in the development of products. For data collection was used a thermal camera Flir EX6 with focal plane array detector (FPA) and uncooled microbolometer with spectral range from 7.5 to 13 micrometers. For body samples were selected polymers PP, PMMA, polystyrene, PU, PVC and expanded rigid, $P A$ and $P C$ in size of $80 \times 80 \mathrm{~mm}$ and thicknesses ranging between 3 and $5 \mathrm{~mm}$. The variation in thickness is due to the industry standard. The study included 10 people, men and women, of several ages, in not-stratified sample. With this study, it is intended to confirm the thermography as an assistant tool for research, for better interpretation of thermal variables of polymers, and to determine both: the contact sensitivity to the conductivity of each material, and if the thermal conductivity factor alters the perception of its qualities by users.
\end{abstract}

Keywords: thermography, thermogram, perception of materials, design, polymers.

\title{
1. Introdução
}

Devido à evolução dos processos de representação e de comunicação de resultados de projeto, torna-se necessário ampliar e incorporar diversas outras possibilidades tecnológicas, principalmente aquelas que carregam inovações para o processo de produção de objeto. Assim, a ferramenta termográfica possibilita ao designer reunir recursos adicionais aos procedimentos de mapeamento da relação de percepção, permitindo uma prospecção aprofundada e interior da matéria.

As aplicações da termografia em processos e procedimentos de projeto de produtos tem-se mostrado crescente em decorrência do "barateamento" da tecnologia empregada nas termocâmeras existentes no mercado. Em suas diversas aplicações, atualmente, intensificou-se sua aplicação na geração de informações a respeito do corpo humano; na interface de contato com os objetos por transferência de 
calor; na avaliação das propriedades de um material; para identificação de pontos de atrito a partir da geração de calor de cada material empregado no objeto; na avaliação de materiais a partir do interior dos produtos, onde é imprescindível a qualidade técnica de produção, objetivando sanar ou minimizar riscos.

Considerando a importância da termografia, este artigo direciona esforços para validar esse instrumento de coleta auxiliar de dados, que permite compreender a percepção a partir da interação e de estímulos do usuário com polímeros. Averiguando, assim, tanto a sensibilidade de contato, como a condutividade de cada material, além de determinar se o fator de condutividade térmica altera a percepção de qualidade do material pelos usuários. E, desse modo, através do processo de mapeamento, identificar qualidades inerentes a cada tipo de material e indicar novas possibilidades e alternativas de seu uso.

Neste sentido, a ferramenta apresenta-se como um recurso relevante para captação de dados térmicos, auxiliando na captura de dados, os quais poderão se tornar diferenciais e indutivos para o processo de projeto. É uma ferramenta de diagnóstico rápido, habilitado a gerar termogramas que auxiliam na interpretação de variáveis térmicas através de software específico. Constitui-se em um equipamento de verificação in loco do comportamento dos materiais, promovendo a detecção da condutividade térmica. Também, poderá auxiliar na análise da percepção tátil do usuário, identificando qualidades inerentes a cada tipo de material, além de indicar novas possibilidades e alternativas de aplicação, devido à composição de fabricação do polímero, que é modificado de fabricante para fabricante.

Essa ferramenta também gera informações a partir do contato e interação do corpo humano com os objetos, por meio do mapeamento das transferências de calor emanadas dessas relações. A termografia apresenta-se como ferramenta auxiliar em uma investigação que busque melhor interpretação das variáveis térmicas dos polímeros. Dessa maneira, busca averiguar tanto a sensibilidade de contato, como a condutividade de cada material e, se, esse fator de condutividade térmica altera a percepção de suas qualidades pelos usuários.

O objetivo do estudo é avaliar por meio do uso da termografia se o nível de transferência de calor realizada pelo usuário em interação com o material plástico, associado a produtos, compromete a percepção das qualidades do produto, interferindo no seu conforto e usabilidade. Propõe-se responder à questão central da pesquisa - como as mudanças térmicas, ocorridas a partir da interação do usuário com o material plástico, aplicado a produtos, podem afetar a percepção das qualidades do material?

\section{Utilização do termograma em projetos de produto}

A termografia é um procedimento de medição passivo e sem contato, que apresenta a distribuição de temperatura na superfície de um objeto. É uma ferramenta que se apresenta como aporte para análises de condutividade térmica e percepção tátil, dentre tantas possibilidades. Destacam-se as informações geradas a partir do contato e interação do corpo com o objeto, através do mapeamento da transferência de calor. Permite, assim, obter estudos criteriosos de materiais e dos acabamentos dos objetos, contribuindo, também, para os estudos a respeito da percepção das qualidades dos materiais na relação usuário-produto.

O objeto em estudo representa um espectro de cores que exprime variações de temperatura do material, detalhes imperceptíveis visualmente. Para os designers, esta ferramenta possibilita ampliar a visão perante os objetos e mapear a relação de percepção, permitindo, assim, uma prospecção mais aprofundada da matéria em estudo. O processo realizado pela ferramenta é constituído pela captura da radiação térmica infravermelha emitida pelo movimento vibratório das moléculas que compõem os materiais. Converte a energia do comprimento de onda infravermelha em imagens de luz visível, onde os objetos, acima do zero absoluto, emitem energia infravermelha térmica. 
Desta forma, a criação da imagem térmica (termograma - em escala policromática, em escala monocromática, ou entre tantas outras possibilidades hoje presentes através do software de tratamento) proporciona, através de uma imagem gerada pela ferramenta, a visualização de áreas e variações de emissão de calor da superfície do objeto. Esta emissão de calor conhecida como emissividade ou poder emissivo, segundo Pereira (2013), é a capacidade de envio de energia por radiação da superfície, relação existente entre a energia irradiada por um corpo real em relação a um corpo negro (objeto ideal que absorve, em qualquer comprimento de onda, toda a radiação incidente sobre ele) sob a mesma temperatura.

As informações obtidas pelo termograma contribuem para uma melhor interpretação das variáveis térmicas dos materiais e ainda colaboram para verificar a sensibilidade de contato realizado. Ainda, o termograma permite a manipulação da imagem, executada por meio de softwares que geram diversas informações gráficas e dados para um mapeamento preciso. A compreensão da emissividade de polímeros é muito relevante para as pesquisas na área, devido à exatidão proporcionada pela captura dos termogramas, pois corpos com altas taxas de emissividade irradiam mais energia que aqueles com baixas taxas, sendo que a quantidade total de radiação depende de sua temperatura e de sua emissividade.

Assim, para este estudo, será necessário o cálculo preciso da emissividade de cada material utilizado na análise de cada amostra. Objetiva-se a construção de uma tabela com os dados de emissividade de cada material coletado, a fim de servir de orientação no ajuste da emissividade da termocâmera. Tal tabela, que será imprescindível, servirá ao processo de captura ao obter exatidão nas imagens térmicas. Desse modo, a criação de tal tabela faz-se necessário para futuros estudos, variando devido à composição de cada polímero.

Para que a termocâmera realize o cálculo da temperatura, outros fatores devem ser considerados, além da emissividade. Fatores que também influenciam na medição, como a transferência de calor, que é dividida em três principais modos - condução, convecção e radiação. Esses processos indicam o estado e a direção da energia transferida. A condutividade igualmente é outro fator de transferência de calor, por meio do qual ele é transferido de um corpo sólido ou pelo fluido em repouso.

Outros fatores atuantes são a reflexão do objeto e ascondições metereológicas durante a captura. Também, os sistemas de calefação e ventilação, sendo que outros aspectos fazem parte da linguagem fotográfica: a nitidez da imagem, a distância focal com relação ao objeto, medições de exposição correta, entre outros detalhes específicos que serão investigados em razão da utilização da termocâmera específica para a coleta de dados (SILVA; TARRALI E MELZ, 2015).

\section{Percepção das qualidades do material plástico pelos usuários}

Entender como pensam os indivíduos que utilizam objetos plásticos em seu contexto de uso e práticas é importante para compreender como se processa a experiência do usuário, sendo que essa compreensão pode se tornar fonte de possíveis inovações e melhorias na experiência com os produtos e materiais.

Os significados de materiais atribuídos pelos usuários, em um contexto particular, são consequência da interação entre usuário-produto e material. Os materiais de que são feitos os produtos afetam a atribuição de significados aos mesmos, conforme relatado por diversos estudiosos afetos a esse campo: Krippendorff e Butter (2007); Ashby e Johnson (2011); Schiffeirstein e Hekkert (2008); Karana e Hekkert (2010); Karana, 2010 (apud MIRA, 2015).

Os significados conferidos aos materiais pelos seus usuários só são compreensíveis quando da interação com produtos em sua vida diária. Esses significados podem ocorrer em contextos distintos, e variar entre 
diferentes indivíduos e culturas. Além disso, significados variam com o tempo e de acordo com a memória de experiências anteriores ou associações (GIBSON, apud HOCHBERG, 1994). Dependendo do contexto de uso, as funções práticas dos objetos podem conter mais de um significado e variar em diferentes contextos.

Durante o processo de interação, os indivíduos utilizam-se de todos os sentidos. Ficou evidenciado na pesquisa referenciada (MIRA, 2015) que a aparência estética, notada pelo usuário, cria a expectativa inicial, que leva a um segundo momento de avaliação - a interação táctil. Isto porque a estética de um produto trabalha criando a expectativa, os atributos visuais operam estimulando os sentidos da visão e emoção; ao passo que é por meio dos outros sentidos, principalmente o táctil, que o usuário avalia o material, experimenta produto e material e elabora seus significados. Logo, este nível de percepção é decisivo para a decisão do usuário.

A percepção estética dos materiais significa os atributos que relacionamos por meio dos sentidos - visão, tato, audição, olfato e paladar. "A estética desperta o interesse, estimula e atrai os sentidos; materiais plásticos podem ser tão macios ao toque quanto tecidos e resistentes como o metal, mas são quentes ao tato", na visão de Ashby e Johnson (2011). A estética é também entendida pelo usuário como sendo belo ou não; pode ser a aparência da forma, as cores da moda ou uma textura que confere à superfície um toque agradável; pode ser ainda uma maneira de expressar uma mensagem sociocultural, um estilo por meio da expressão da forma e do material, de acordo com Muller, citado por Ross e Wensveen (2010).

Certas características, que podem ser avaliadas por meio do sentido da visão, podem ser confirmadas ou não através do toque. Utiliza-se a percepção táctil para a avaliação de formas, qualidades de materiais, texturas, suavidade, temperatura, flexibilidade, resistência, peso, dentre as muitas características de um produto, além de fornecer referência e permitir os acionamentos, tais como montagem e desmontagem, limpeza e manutenção de produtos (FAUCHEAU et al, 2015, p. 1-2).

Plásticos representam uma categoria de materiais à parte. São versáteis, possuem propriedades demandadas e ajustadas a muitos segmentos de mercado, e a milhares de produtos estão associados. Para cada tipo específico de produto é possível encontrar um polímero adequado àquela aplicação. Quando designers e produtores necessitam de um esforço ampliado, objetivando melhoria de desempenho, ou uma característica que se associe à funcionalidade de um produto, é possível alterá-lo modificando sua composição, adicionando compostos ou cargas ou combinando-o com outros polímeros (blendas poliméricas), que reforçam uma determinada propriedade, a qual se pretende ampliar e representam um universo de possibilidades, pois mimetizam outros materiais.

A fim de aprofundar conhecimentos acerca das variáveis que interferem na experiência com materiais, os dados coletados por meio desta investigação - a emissividade e a condutividade térmica dos materiais, poderão ser de relevância para entender como se processa a percepção e os estímulos ou não desta variável e como a composição dos polímeros altera esses dados. Sendo assim, há a necessidade de aferir procedimentos, criar parâmetros por meio de tratamento e organização dos dados, que servirão para obtenção de informação de qualidade e contribuirá para o campo do design, como recurso para o desenvolvimento e melhoria de produtos e da interação de usuários com eles. Desse modo dando ênfase à necessidade de detalhar os processos e/ou procedimentos adotados para a coleta de dados. 


\section{Método}

\subsection{Considerações a respeito do método}

Objetivando aprofundar os conhecimentos a respeito da percepção dos usuários, relacionada aos materiais plásticos; tendo como base os resultados obtidos a partir da pesquisa referenciada (MIRA, 2015) e, também, objetivando testar um instrumento de coleta de dados quantitativo - a termografia, passa-se a descrever os procedimentos do método aplicado.

Foram convidados a participar do estudo dez participantes, entre homens e mulheres, em amostra não estratificada. Explicou-se a cada um dos participantes os procedimentos que ocorrem no ambiente preparado para o experimento, detalhando, primeiramente, o uso do equipamento, o ambiente preparado e o modo do procedimento de contato com as amostras (primeira, segunda e terceira etapas). Após todas as considerações, mediu-se a temperatura da amostra no ambiente, a qual foi anotada na ficha de análise de cada participante, ficha essa formulada pelos pesquisadores. Na etapa seguinte, mediu-se a temperatura das mãos de cada participante, em estado de repouso, e realizado o segundo termograma.

Em seguida, cada participante foi orientado a tocar, apenas com o dedo indicador, o corpo de prova, em uma inspeção cega, durante dez segundos (amostra delimitada pelo tamanho de 80x80 mm). Realizou-se o terceiro termograma, onde o participante não teve contato visual com a amostra. Ato contínuo, foi solicitado ao participante que interagisse mais com a amostra, momento em que foi aferida a transferência térmica. Nessa etapa foi possível identificar a quantidade de calor emitido pela superfície da mão após a manipulação da amostra. Assim, para esta análise, o participante interagiu com o corpo de prova por 10 segundos, a fim de atingir um equilíbrio térmico com o ambiente. Após este período, procedeu-se à captura térmica.

\subsection{Diretivas para a captura termográfica e uso do equipamento}

Os dados discriminados abaixo servem como orientação e parâmetro, os quais foram mantidos durante cada coleta e verificados constantemente para uma obtenção precisa dos termogramas, seguindo as orientações do manual da ferramenta Flir (2015).

Antes de realizar a captura com a termocâmera, procedeu-se à limpeza da lente infravermelha, a fim de obter um resultado preciso durante as etapas de coleta de dados, sem interferências. Também foi realizado uma limpeza do corpo de prova após cada coleta realizada e mantendo um intervalo para cada coletada para não interferer na temperatura da amostra. Para se obter resultados precisos, o fabricante recomenda que se aguarde cinco minutos após ter iniciado a câmera para realização da captura, o que foi realizado. Outro ponto é a mudança da paleta de cor (ferro), selecionada pelo sistema da termocâmera, que será necessária para facilitar a análise da imagem durante o processo de captura.

Por se tratar de análise de imagem, foi necessário verificar os seguintes parâmetros: a quantidade de energia infravermelha emitida, transmitida e refletida pelo objeto; o sistema previamente selecionado; a imagem dinâmica multiespectral (MSX), onde a termocâmera apresenta uma imagem infravermelha em que os contornos da amostra ficam mais definidos. Esse procedimento possibilitou definir a distância de alinhamento entre a termocâmera e a amostra. Procedeu-se à realização de imagens de forma tradicional para registro de comunicação dos dados e documentação de todas as etapas de coleta.

A distância entre a câmera e corpo de prova é um fator importante para obtenção de maior confiabilidade dos dados e precisão do registro. Requer que sejam definidos previamente e que seja utilizado o mesmo critério de valor para todas as capturas realizadas, ainda que com mudança das amostras. Assim, a termocâmera permaneceu fixa para que a seleção dos medidores de ponto fossem travados de forma a não 
ocorrer mudanças. Tal parâmetro foi utilizado para compensar a radiação que é absorvida pela atmosfera e a radiação da própria atmosfera.

Outro aspecto que foi levado em consideração foi a compensação da umidade relativa presente na atmosfera, sendo imprescindível definir a umidade relativa para o valor apropriado. Para distâncias de captura reduzidas, como a estipulada no estudo, adotou-se as recomendações técnicas do manual do equipamento (FLIR, 2015, p.58), definindo-se um valor de 50\% - Neste modelo utilizado, já é definido automaticamente essa taxa para a umidade. A ferramenta também permitiu compensar o parâmetro de temperatura atmosférica entre a câmera e o objeto - alvo para $24^{\circ} \mathrm{C}$, temperatura mantida no ambiente climatizado.

É importante frisar que a precisão da termocâmera utilizada neste trabalho gerou um erro de captura, considerado um fator de variação na coleta de dados desta pesquisa, característica comum a outras análises que utilizam essa ferramenta. $\mathrm{O}$ erro de precisão do equipamento Flir Ex6 pode variar de $\pm 2^{\circ} \mathrm{C}$ $\left( \pm 3,6^{\circ} \mathrm{F}\right)$ ou $\pm 2 \%$. Assim, a cada coleta é necessário ligar e desligar o equipamento, aguardando o tempo de repouso recomendado pelo fabricante, como mencionado anteriormente, para obtenção de melhores resultados.

\subsection{Definindo o grau de emissividade de materiais personalizados}

Segundo o manual (FLIR, 2015) do equipamento utilizado, a "emissividade" é uma propriedade que indica a quantidade de radiação gerada por um objeto comparada com a quantidade refletida pelo mesmo. Para uma medição térmica precisa, definiu-se o grau de emissividade para cada amostra, determinando padrões para inserção da ferramenta. O modelo de termocâmera utilizado neste estudo foi a Flir Ex6, que possibilita variáveis de 0,1 a $1,0 \varepsilon$. Considerou-se que há emissividade da planta das mãos em repouso e durante o experimento foi fixado um valor de $0.98 \varepsilon$, conforme tabela fornecida em forma de aplicativo (Infrared Emissivity table for android) pelo fabricante do equipamento.

A termocâmera utilizada, bem como outros registros documentais sobre o uso da ferramenta, não registra a emissividade de polímeros. Logo, foi necessário identificar um valor personalizado para cada material analisado, a fim de obter medições mais precisas. Sendo assim, antes da realização da coleta de dados, foi imprescindível realizar o cálculo de emissividade para cada amostra, seguindo orientações do fabricante.

A radiação refletida pelo objeto foi compensado quando necessário. Havendo baixa emissividade e temperatura do objeto significativamente diferente da temperatura aparente refletida, definiu-se a compensação, ajustando corretamente a temperatura aparente refletida no polímero. Neste caso, a interface do equipamento utilizado - Flir Ex6 permitiu ser ajustado automaticamente, com base na entrada da temperatura refletida.

\subsection{Variáveis consideradas}

Foram considerados como amostras de materiais polímeros laminados encontrados no mercado, que também coincidem com os materiais aplicados ao design de produtos. Para definição dessas amostras, foram considerados critérios de seleção de materiais coincidentes com o aplicado ao design de produtos de uso pessoal e/ou doméstico. Em algumas categorias de produtos, o material aplicado não foi encontrado na forma laminada e por essa razão não fez parte das amostras para o experimento, como por exemplo o polímero melamina. Assim, considerou-se variáveis importantes, observadas na medição das amostras e descritas a seguir: amostra plana sem textura, amostra com textura e com brilho, amostra com textura e sem brilho, geometria do material, diferentes materiais plásticos e temperatura em repouso e após o contato. 


\subsection{Seleção dos materiais}

Foram selecionados para esse estudo polímeros que são encontrados no mercado sob a forma de chapas planas. Em sua maioria commodities, pois são esses utilizados com maior frequência nos produtos, sendo eles: PMMA, termoplástico com alto índice de transparência; Poliestireno, termoplástico commoditie de uso geral; PP, termoplástico commoditie; PS, termoplástico/poliolefinas; PVC rígido; PA ou Nylon 6.6, polímero de engenharia; PVC expandido. Todas as amostras eram planas e possuíam o mesmo tamanho (80x80mm), variando apenas na espessura ( 3 a $6 \mathrm{~mm})$. Apenas o nylon apresentava textura diversa, que é inerente à própria característica do material.

Cada polímero, de acordo com suas propriedades e características, decorrentes de suas composições, mantém condutividade térmica diferente e que será possível de identificar atráves do estudo. As análises identificaram variáveis de retenção de calor resultante do contato, revelando quais amostras reteram maior quantidade de calor e se possuíam menor condutividade térmica.

\subsection{Procedimentos para a coleta de dados}

Para realizar a coleta de dados de forma precisa foi necessário adotar os procedimentos e recomendações disponibilizados no manual da ferramenta - FLIR. Manual do utilizador série Flir Ex (2015).

Delimitou-se realizar aferições relacionadas à condutividade térmica, a fim de correlacionar esses valores aos resultados obtidos na pesquisa de Mira (2015) sobre a qualidade dos materiais, na visão dos usuários. Por ser a condutividade térmica uma variável, era possível sua interferência nos resultados, tendo em vista que alguns grupos de polímeros possuem um alto nível de emissividade térmica. Logo, para controlar o ambiente onde foram realizadas as capturas, foi desenvolvido um aparato para o estudo. a fim de facilitar o mapeamento junto aos dez participantes. Fixou-se a termocâmera neste aparato a fim de manter a mesma distância durante o registro de cada coleta, conforme apresentado nas figuras 1 e 2 .

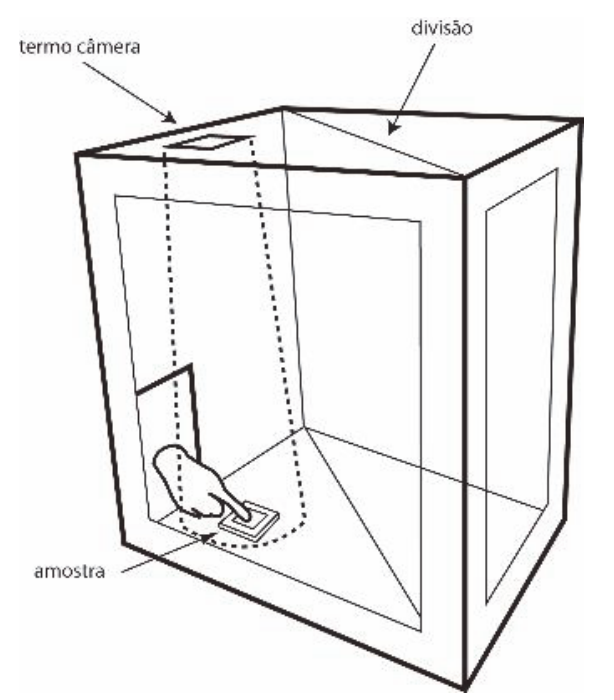

Fig. 1 Diagrama do aparado desenvolvido para a coleta de dados 


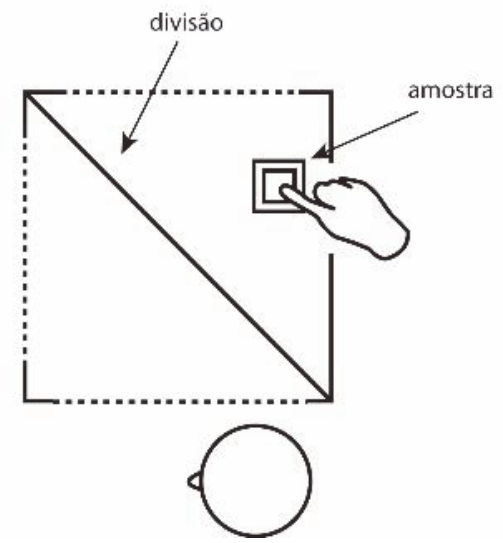

Fig. 2 Diagrama do aparado desenvolvido para a coleta de dados

$\mathrm{O}$ aparato possibilitou a troca de amostras, e posicionando-o corretamente, foi possível também que o participante do estudo não visualize o objeto alvo da captura. Procedeu-se dessa maneira para que o estímulo visual não interferisse no resultado da coleta, bem como para garantir o emprego do método proposto. Conforme demonstra imagem a seguir figuras 3 e 4 .

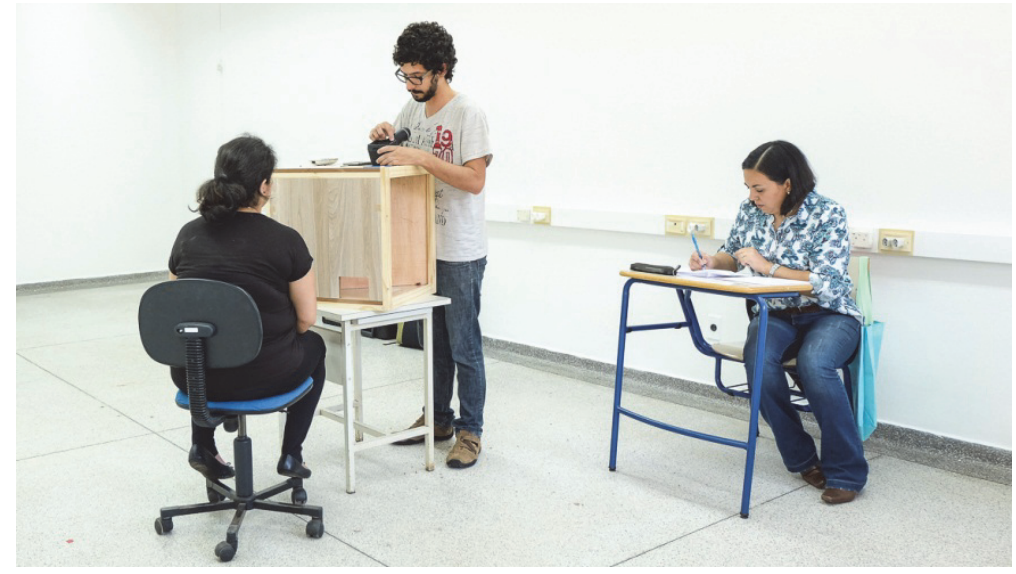

Fig. 3 Coleta de dados no Laboratório de Ergonomia e Interfaces (LEI). Foto: Ana Paula Maldonado

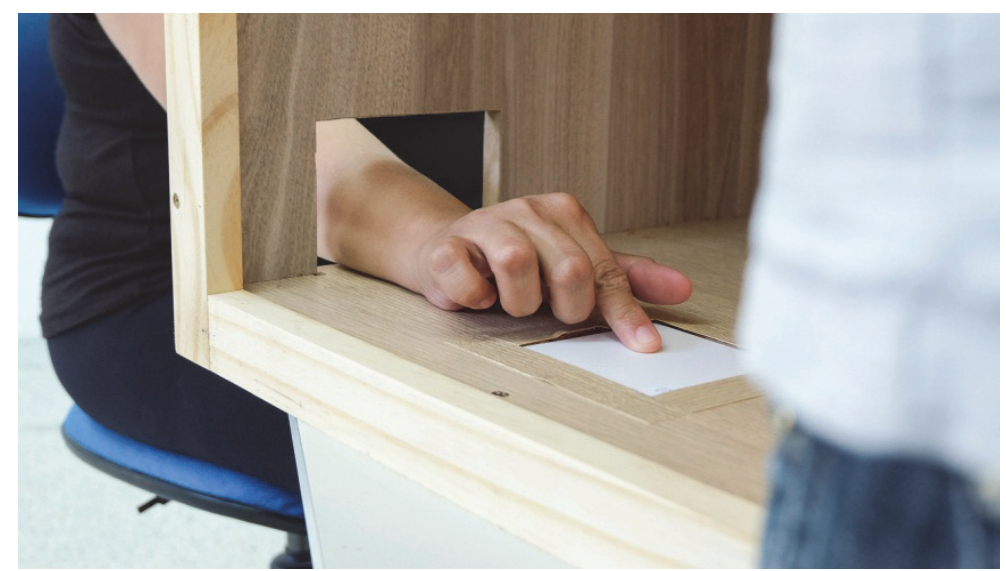

Fig. 4 Coleta de dados no Laboratório de Ergonomia e Interfaces (LEI). Foto: Ana Paula Maldonado 
Inicialmente, mapeou-se a amostra em repouso no ambiente, considerando a umidade e a temperatura, dados esses que foram registrados. Após esse procedimento, aferiu-se a temperatura da planta da mão do participante, sem contato com a amostra. A partir da realização desses procedimentos, que antecedem o contato com a amostra do material, é que foi realizado o mapeamento do contato do participante em ambiente controlado para obtenção da condutividade térmica. O contato foi mantido por dez segundos, cronometrados em todas as coletas, para todas as amostras.

A condutividade térmica é uma propriedade física dos materiais, motivo pelo qual todos os materiais possuem a capacidade de conduzirem calor (CALLISTER, 2002). O fluxo de calor ocorre por condução, convecção ou radiação, conforme mencionado nas diretivas para a captura termográfica. Segundo Dischinger (2009) um material será percebido como frio ao toque se ele conduzir rapidamente o calor recebido pelo dedo. Para identificar a disbribuição de temperatura das amostras foi necessário realizar a coleta de dados em um ambiente climatizado e com temperatura ambiente controlada.

Solicitou-se, também, que os participantes registrassem suas impressões em um formulário préformatado, no qual constava um código de identificação para cada amostra. Nessa parte do registro de impressões o usuário poderia manipular o corpo de prova ampliando o contato. Não se estimulou a resposta dos participantes por meio de atributos pré-definidos, tendo em vista a busca por uma descrição mais espontânea.

\subsection{Tratamento dos dados coletados}

Como forma de representação, essa ferramenta visa facilitar a interpretação de dados invisíveis à primeira vista, pois a olho nu eles não são perceptíveis. $O$ uso desse instrumento de representação visual facilita, também, o desenvolvimento e análise de materiais, podendo contribuir para a concepção e escolha de materiais mais adequados à produção de objetos.

Para tratamento dos dados, depois de coletados, foram indexados e realizados procedimentos de análise considerando a emissividade do material e a transferência de temperatura do participante para a amostra de material plástico. Considerou-se as variáveis importantes já descritas anteriormente (item 3.4).

A termocâmera (figura 5) utilizada pertence ao Laboratório Didático de Conforto Ambiental (LADICA), do Departamento de Arquitetura, Urbanismo e Paisagismo da FAAC/UNESP e a análise das termografias foi enpreendida com o uso do software Flir Tools. A coleta de dados foi realizada no Laboratório de Ergonomia e Interfaces (LEI) do mesmo departamento da Universidade Estadual Paulista (UNESP), em sala climatizada, cujo termômetro indicava a temperatura de $24^{\circ} \mathrm{C}$. 


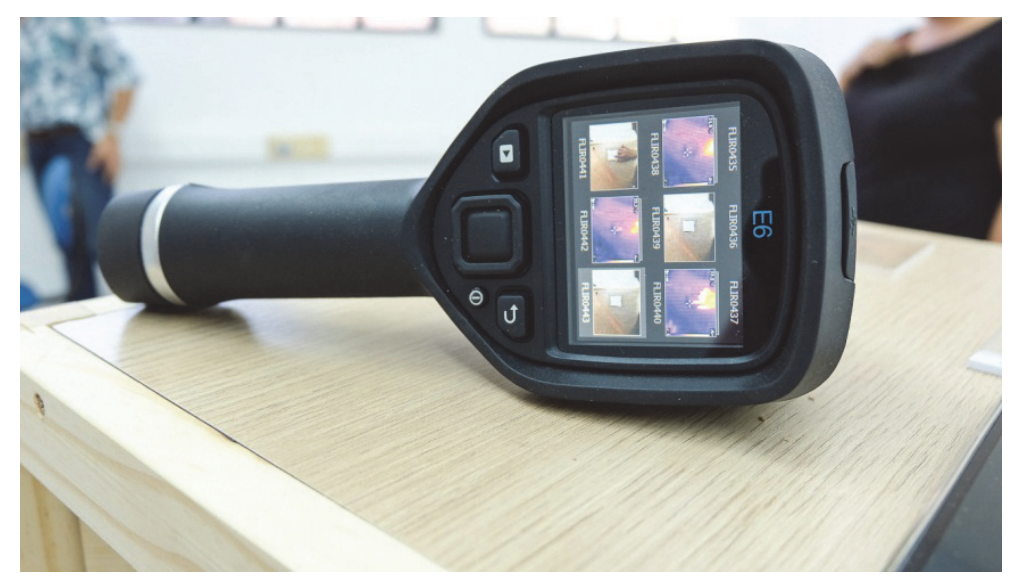

Fig. 5 Termocamera Flir EX6. Foto: Ana Paula Maldonado

\section{Resultados}

Em cada termograma obtido foi realizada uma leitura pelo software mencionado, por meio do qual foi possível visualizar a temperatura exata de cada ponto da amostra, através de um ponto de medição preciso. Outra ferramenta associada ao software permitiu traçar uma faixa de medição, que possibilitou identificar as temperaturas máxima, mínima e média de cada amostra, assim como a função delta do ponto de contato. Assim foi possível observar a variação de temperaturas, através da condutividade de cada material, conforme pode ser observado nas figuras 6, 7, 8, 9 e 10 do participante p1t. Identificando diferenças da difisuidade do calor em amostras com alto e baixo índice de condutividade térmica.

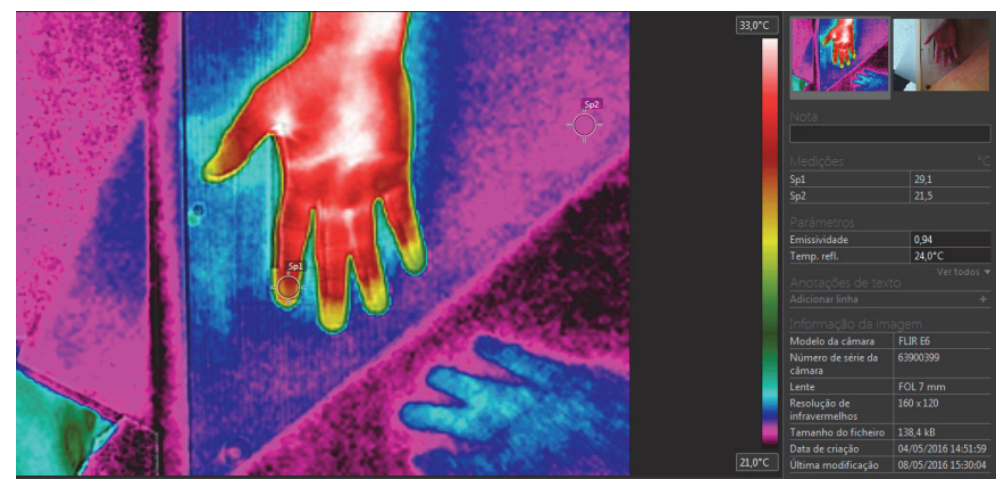

Fig. 6 - Termograma da temperatura da mão em repouso $-29,1^{\circ} \mathrm{C}$ 
Termografia: Una ferramenta auxiliar na pesquisa de materiais e no design de produtos.

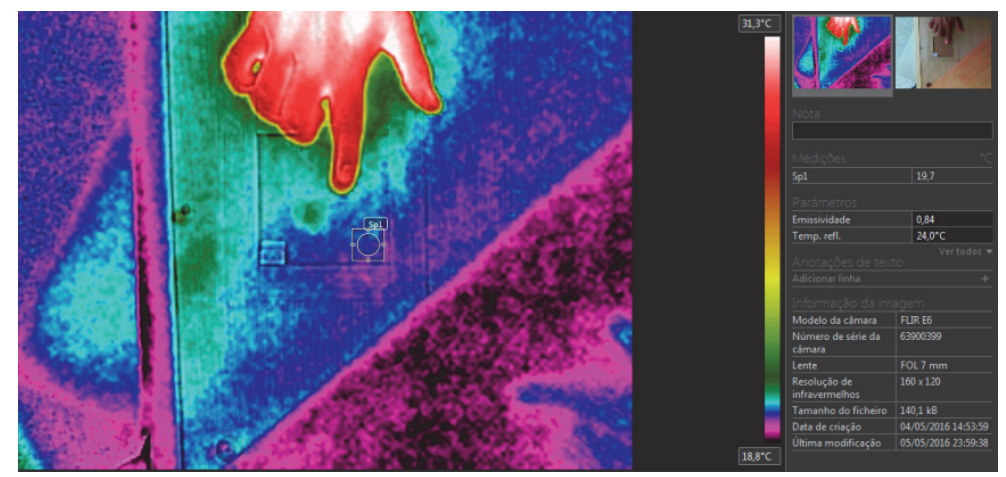

Fig. 7 Termograma da temperatura da amostra com o contato inicial do polegar com a amostra PMMA $-19,7^{\circ} \mathrm{C}$

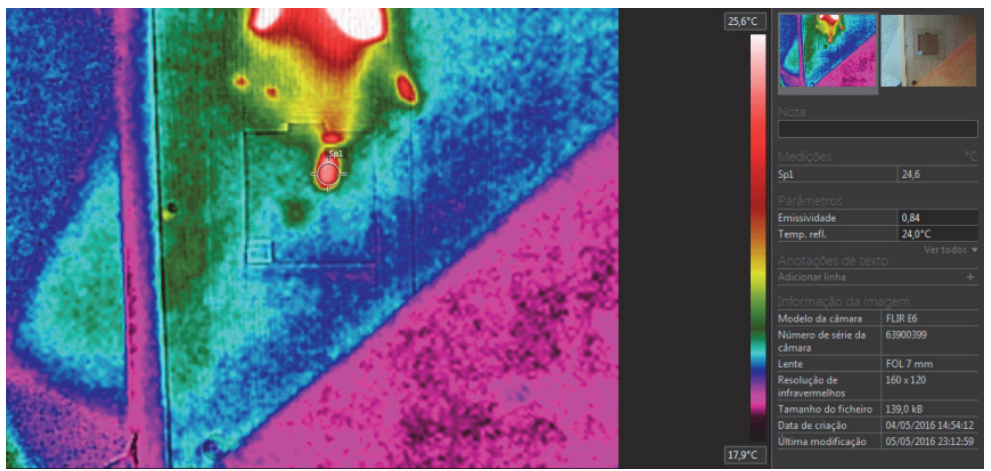

Fig. 8 Termograma apos 10 segundos de contato do polegar com a amostra PMMA -24,6 $6^{\circ} \mathrm{C}$

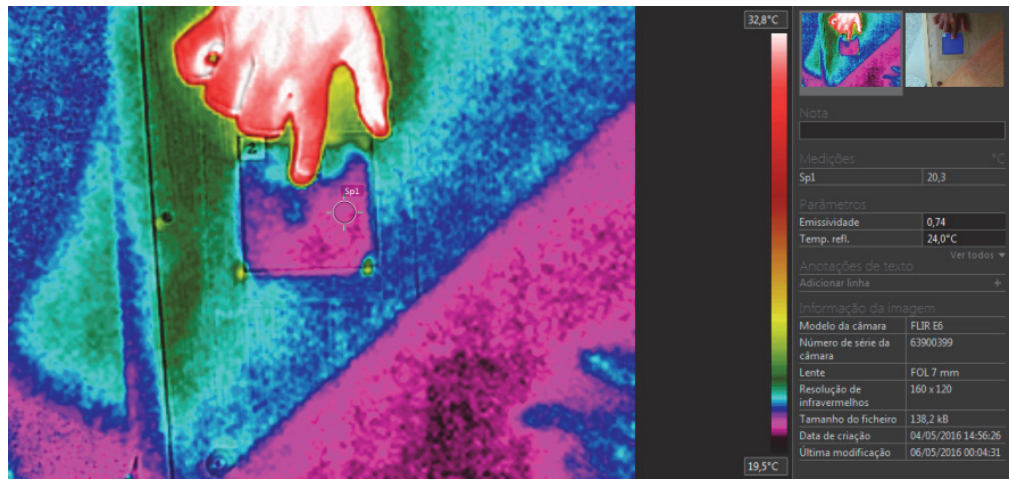

Fig. 9 Termograma da temperatura da amostra com o contato inicial do polegar com a amostra Poliestireno $-20,3^{\circ} \mathrm{C}$

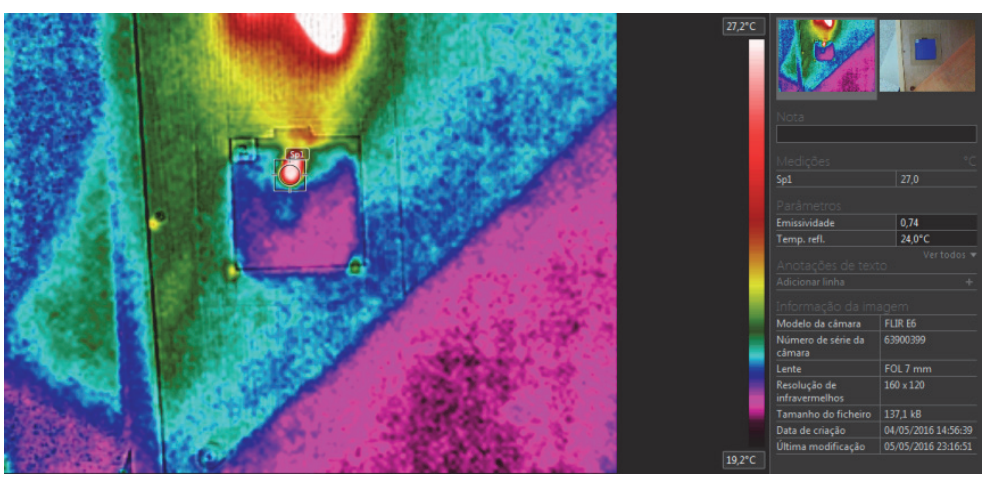

Fig. 10 Termograma após 10 segundos de contato do polegar com a amostra Poliestireno - 27, $0^{\circ} \mathrm{C}$ 
Por meio da leitura das imagens termográficas de forma ampla, com ênfase na analise de toda a coleta, foi possível verificar que a temperatura obtida de cada participante (ponto de leitura no dedo indicador) manteve-se, em média, aproximadamente em torno dos $4,5^{\circ} \mathrm{C}$ de diferença da temperatura ambiente $\left(24^{\circ} \mathrm{C}\right)$. No aparato, durante todo o experimento, manteve-se a temperatura de $0,55^{\circ} \mathrm{C}$ de diferença em relação ao ambiente $\left(24^{\circ} \mathrm{C}\right)$, preservando uma média de temperatura em torno dos $23,45{ }^{\circ} \mathrm{C}$. Os pesquisadores não verificaram que essa pequena alteração de temperatura do aparato tenha interferido nos resultados obtidos ao logo do experimento.

Houve variações com relação às amostras, conforme tabela 1 abaixo:

Tabela 1. Estratificação dos dados do participante p1t

\begin{tabular}{|c|c|c|c|c|c|c|c|c|c|}
\hline P1t & Polímeros & ambiente & temp. mãos & temp. amostra s/c & no. captura inicial & no. captura final & tempo contato & difusividade & condutividade térmica \\
\hline & PMMA & $24^{\circ} \mathrm{C}$ & 29,1 & $20^{\circ} \mathrm{C}$ & 19,7 & 24,6 & $10 \mathrm{~s}$ & 4,9 & $0,08-0,25 \mathrm{~W} / \mathrm{m}^{*} \mathrm{~K}$ \\
\hline & Poliestireno & & & $20,7^{\circ} \mathrm{C}$ & 20,3 & 27,0 & $10 \mathrm{~s}$ & 6,7 & $0,12-0,13 \mathrm{~W} / \mathrm{m}^{*} \mathrm{~K}$ \\
\hline & PP & & & $21,9^{\circ} \mathrm{C}$ & 22 & 27,2 & $10 \mathrm{~s}$ & 5,2 & $0,11-0,17 \mathrm{~W} / \mathrm{m}^{*} \mathrm{~K}$ \\
\hline & PA & & & $22,1^{\circ} \mathrm{C}$ & 22,3 & 27,8 & $10 \mathrm{~s}$ & 5,5 & $0,18-0,35 \mathrm{~W} / \mathrm{m}^{*} \mathrm{~K}$ \\
\hline & PVCex & & & $22,4^{\circ} \mathrm{C}$ & 22,6 & 30,9 & $10 \mathrm{~s}$ & 8,3 & $0,15-0,29 \mathrm{~W} / \mathrm{m}^{*} \mathrm{~K}$ \\
\hline & PVCrig & & & $22,0^{\circ} \mathrm{C}$ & 22 & 29,6 & $10 \mathrm{~s}$ & 7,6 & $0,15-0,29 \mathrm{~W} / \mathrm{m}^{*} \mathrm{~K}$ \\
\hline & PC & & & $22,5^{\circ} \mathrm{C}$ & 22,5 & 29,1 & $10 \mathrm{~s}$ & 6,6 & $0,19-0,22 \mathrm{~W} / \mathrm{m}^{*} \mathrm{~K}$ \\
\hline
\end{tabular}

Observou-se, através dos dados obtidos por meio dos termogramas, que as amostras PMMA (Resarbrás / Unigel), PA (Plastireal), PC (Bayer) e PP (Durplastic) não concentraram tanto calor através do toque quanto as amostras PVCrig e PVCex (Rochling) e o Poliestireno (Golden Signs). Percebeu-se que entre todas as amostras mapeadas, aquelas com maior retenção de calor também apresentaram menor condutividade térmica, haja vista que o calor aplicado sobre elas não se dissipou rapidamente.

Outro aspecto a ser esclarecido é que as medidas indicadas pela termocâmera, conforme mencionadas nas diretivas da coleta, não sao absolutas e que em estudos mais aprofundados se faz necessário a utilização de uma termocâmera que possua melhor qualidade de captura. Da mesma forma, se faz necessário ressaltar que para estudos futuros é imperioso mapear a interação com os produtos, a fim de verificar alguns tópicos relevantes, como por exemplo se a condutividade térmica do material afeta a percepção de qualidade do produto.

Fica evidente que a composição do polímero tem influência na condutividade do material, motivo pelo qual é possível compreender a razão pela qual a literatura existente sobre a condutividade térmica fornece apenas dados variáveis, tendo em vista que não levam em conta as características de cada fabricante. Para que o designer obtenha melhores resultados, com diferentes tipos de polímeros, é necessário que o fornecedor apresente tais dados.

\subsection{Considerações finais sobre os resultados obtidos}

Considerando a importância da termografia, este artigo direciona seus estudos para validar esse instrumento auxiliar de coleta de dados, que permite compreender a percepção a partir da interação do usuário com polímeros, identificando qualidades inerentes a cada tipo de material e indicando novas possibilidades e alternativas de uso.

Tem em conta que dados mais precisos de condutividade e emissividade térmica são relevantes para a aplicação do material e para os aspectos que se associam à percepção dos usuários, sugere-se a ampliação 
desse estudo. Concluiu-se que os materiais plásticos podem ser quentes ou frios, razão porque seu índice de condutividade térmica é determinante em muitas de suas aplicações, sendo que a capacidade de conduzir calor, sem dúvidas, pode afetar a experiência com o produto e com os materiais. Como exemplo pode-se citar os polímeros associados a produtos eletrônicos, que necessitam possuir isolantes térmicos e acústicos. Ainda, é possível mencionar os polímeros associados aos eletrodomésticos que precisam produzir calor ao processar alimentos, sem que isso represente perigo aos usuários. Em síntese, esse estudo não esgota resultados, fazendo-se, ainda, necessário empreender estudos complementares que possam ir além dos resultados aqui apresentados.

\section{Agradecimentos}

Os autores agradecem ao $\mathrm{CNPq}$, pela concessão da bolsa de doutorado (ao primeiro autor); aos professores Prof. Dr. João Roberto Gomes de Farias, por fornecer a termocâmera pertencente ao Laboratório Didático de Conforto Ambiental (LADICA), do Departamento de Arquitetura, Urbanismo e Paisagismo da FAAC/UNESP e ao Prof. Dr. Luis Carlos Paschoarelli, por permitir o uso do espaço climatizado do Laboratório de Ergonomia e Interfaces (LEI) do mesmo departamento da Universidade Estadual Paulista (UNESP), para realização da coleta de dados.

\section{References}

ASHBY, M. \& JOHNSON, K. (2011). Materiais e design: arte e ciência da seleção de materiais no design de produto. Rio de Janeiro: Elsevier.

CALLISTER JR. W. D. (2002). Ciência e engenharia de materiais: uma introdução. 5. ed. Rio de Janeiro: LTC.

DISCHINGER, M. C. T. (2009). Metodologia de análise sensorial tátil em diferentes classes de materiais e texturas para aplicação no design de produtos, Dissertação (Mestrado) - Universidade Federal do Rio Grande do Sul, Escola de Engenharia e Faculdade de Arquitetura. Programa de Pós-Graduação em Design, Porto Alegre, 166.

FAUCHEAU et al, (2015). Experimental setup for visual and tactile evaluation of materials and products through Napping. Disponível em publication at: https://www.researchgate.net/publication/280935322, p. 1-2.

FLIR. Manual do utilizador série Flir Ex. Wilsonville: Intertek. (2015). 96p. Disponível em: <http://80.77.70.144/DocDownload/Assets/dl/t559828-pt-pt\$ad.pdf>. Acesso em: 12 jan. 2015.

HOCHBERG, J. (1994). James Jerome Gibson: A biographical memoir by Julian Hochberg. National Academy of Sciences Washignton D.C.

KARANA, E (2010). How do materials obtain their meanings? Middle East Technical University (Ankara) Turkey. In: http://jfa.arch.metu.edu.tr/archive/0258- 5316/2010/ciet27/sayi_2/271-285.pdf.

KARANA, E. \& HEKKERT, P. (2010). User-material-product Interrelationships in Attributing Meanings. International Journal of Design, Vol.4, no. 3, 2010. In: www.ijdesign.org/ acesso 7/2/2013.

KARANA, E; PEDGLEY, O \& ROGNOLI, V. (2014). Materials Experience: Fundamentals of materials and design. Oxford: Elsevier.

KRIPPENDORFF; BUTTER. (2007). Semantics: Meanings and contexts of artifacts. Annenberg School for Communication: University of Pennsylvania.

MIRA, M.R.G. (2015). Percepções dos usuários a respeito do material plástico aplicado ao design de produtos. Dissertação de mestrado. Faculdade de Arquitetura e Urbanismo, Universidade de São Paulo, São Paulo, SP, 321p.

MURARIU, A.A.C. et al. (2012) Application of Thermography in Materials Science and Engineering, Infrared Thermography, Dr. Raghu V Prakash (Ed.), ISBN: 978-953-51-0242-7, InTech, Available from: http://www.intechopen.com/books/infrared-thermography/applications-of-thermography-in-materials-scienceandengineering. 
NETO, P.A. (2004). Noções sobre termografia: integridade de equipamentos. São Paulo: Integra, Cooperativa dos profissionais em integridade de equipamentos, $14 \mathrm{p}$.

NOVO, M.M.M. et al. (2014). Fundamentos básicos de emissividade e sua correlação com os materiais refratários, conservação de energia e sustentabilidade. Universidade Federal de São Carlos. Disponível em http://www.scielo.br/pdf/ce/v60n353/04.pdf Cerâmica (60) p. 22-33.

PEREIRA, R. R. (2013). Características térmicas de assento de cadeiras escolares por termografia, Dissertação (Mestrado) - Escola de Design, Programa de Pós-Graduação em Design da Universidade do Estado de Minas Gerais, Belo Horizonte, 91.

ROSS, P. \& WENSVEEN, S.A.G. (2010). Design behavior in interaction: Using aesthetic experience as a Mechanism for design. International Journal of Design, Vol.4, no. 2. In: www.ijdesign.org acesso em acesso em $1 / \mathrm{mar} / 2014$.

SCHIFFERSTEIN, H.N.J. \& HEKKERT, P. (2008). Product experience. San Diego: Elsevier

SILVA, J. C. R. P.; TARALLI, C. H. \& MELZ, S. P. M. (2015). Termograma: a imagem térmica como instrumento de diagnóstico rápido no design. In: IV International Conference on Design, Engineering, Management for innovation, Florianópolis. IDEMI 2015. Florianópolis: UDESC, 2015. v. 1. p. 313-324.

YANAGISAWA, H \& HAKATSUJI, K. (2015). Effects of visual expectation on perceived tactile perception: An evaluation method of surface texture with expectation effect. Disponível em: <www.ijdesign.org>. International Journal of Design Vol. 9 No. 1. Acesso em: 15 jan. 2015. 